Dritte Pflegereform

\section{bpa befürchtet weniger Vielfalt an Angeboten}

— „Wir brauchen in Nordrhein-Westfalen vielfältige und passende Pflegeangebote, dann können die Bürger selbst entscheiden, welche Unterstützung und Versorgung sie wünschen und brauchen." Der Präsident des Bundesverbandes privater Anbieter sozialer Dienste (bpa), Bernd Meurer, hat die im aktuellen Pflegestärkungsgesetz III geplante zentrale Rolle der Kommunen kritisiert. "Kommunen sollen den Bedarf ermitteln und die Rahmenbedingungen für eine entsprechende Versorgung schaffen. In der Vergangenheit haben wir aber vielfach erlebt, wie aus einer Bedarfsplanung bei knappen Kassen schnell eine Steuerung durch Zulassung oder Verhinderung wurde", sagte Meurer auf der bpa-Fachtagung in Neuss. Zuvor hatte sich Gesundheitsstaatssekretärin Martina Hoffmann-Bardache klar für einen breiten Angebotsmix in der Pflege ausgesprochen. Der bpa erwarte aber, dass dieser Mix durch die Landespflegepolitik auch ermöglicht wird, so Meurer. Hoffmann-Bardache warnte angesichts der bevorstehenden Einführung des neuen Pflegebedürftigkeitsbegriffs davor, Verlierer in den Reihen der Pflegebedürftigen zu schaffen. „Für die Menschen, die ab dem kommenden Jahr neu in eine Pflegeeinrichtung kommen, greift die Übergangsregelung nicht mehr. Hier können sich künftig deutlich höhere Eigenanteile ergeben."

www.bpa.de
Junge Pflege

\subsection{0 \\ Jugendliche pflegen Angehörige}

— Rund 1,9 Millionen Pflegebedürftige in Deutschland, die Leistungen aus der Pflegeversicherung beziehen, werden zu Hause versorgt. In gut zwei Drittel wird die Pflege ausschließlich durch pflegende Angehörige geleistet. Auch minderjährige Kinder und Jugendliche unterstützen regelmäßig bei Pflegeaufgaben in der Familie. Etwa 5\% aller Jugendlichen in Deutschland - rund 230.000 - sind dabei bedeutend in die Versorgung Angehöriger eingebunden. Dies zeigt eine repräsentative Befragung der Stiftung Zentrum für Qualität in der Pflege (ZQP) unter 1.000 12 - bis 17-Jährigen.

www.zqp.de

\section{Alles richtig?}

Die korrekten Antworten der PflegeKollegs lauten:

Stoma- und Fistelmanagement

$1 b, 2 a, 3 b, 4 b, 5 a, 6 c, 7 a, 8 b, 9 c, 10 c$

Gewalt am Arbeitsplatz

1a, 2b, 3b, 4c, 5a, 6b, 7c, 8a, 9b, 10b

Vorschau!

Die PflegeKollegs im Monat Dezember:

Allergie oder was?

Gluten - Laktose - \& Co.

Patientensicherheit

Kritisches Ereignis

- CIRS - Sicherheitskultur 